WS7-B02

\title{
The Role of Geomechanics in Reservoir Stimulation Design Procedure
}

\author{
M.M. Slota-Valim (Oil \& Gas Institute) \& H.B. Jedrzejowska-Tyczkowska* \\ (Oil \& Gas Institute)
}

\section{SUMMARY}

Hydraulic fracturing treatment preceded with reliable geomechanical analysis of the reservoir, whether carried out immediately after well completion or during late stages of the reservoir lifetime (refracturing among others), helps to avoid near-wellbore area damage and the hydrocarbons production increase, through the creation of the artificial fracture network and therefore enabling free flow of the gas from the unconventional formation into the wellbore. The practice of stimulation treatment by fracturing the geologic formation reaches 1947, but the early application of hydraulic fracturing was not successful due to the problems with diagnosis of the complications and selection of wells that were about to be subjected to the treatment. Over the last two decades numerous cases of successful secondary fracturing were recorded in the hydrocarbons reservoirs in North America, Russia, China, Brazil and Algeria. Obviously the potential for production increase have hydrocarbon deposits around the world, especially those at advanced stage of exploitation. In most of the cases the use of such treatments is much more economical than determining optimal location, well design, drilling and completion of new well. 


\section{Amsterdam ' 14}

\section{Introduction}

The success of the intensification treatment through hydraulic fracturing in the reservoir rock is dictated by the susceptibility of geologic formation to produce a complex network of artificial fractures. Thus for the proper design of the treatment, the recognition of the factors affecting the brittleness of the rock subjected to the fracturing is crucial.

Whether the rock mass subjected to the pumped under high pressure fracturing mixture behaves in a brittle or a plastic manner, depends on its elastic properties such as Young's modulus (E) [1] or Poisson's ratio (v) [2] (Fjar et al., 2008; Dunphy and Campagna, 2011).

$\mathrm{E}=\sigma / \varepsilon$, where: $\mathrm{E}-$ Young modulus $[\mathrm{Psi} ; \mathrm{MPa}], \sigma-$ stress $[\mathrm{psi} ; \mathrm{MPa}], \varepsilon-$ relative linear strain

$\mathrm{v}=-\varepsilon \mathrm{x} / \varepsilon \mathrm{z}$, where: $\mathrm{v}-$ Poisson's ratio, $\varepsilon \mathrm{x}-$ relative axial strain, $\varepsilon \mathrm{z}-$ relative transverse strain.

The knowledge of the spacial distribution of this parameters combined with the information about the value (magnitude) and direction of the acting stresses allows for hydraulic fracturing design including the geometry of created fractures and the pressure, under which the fracturing fluid should be injected to the target formation (Dozier, 2003; Siebrits et al., 2000).

\section{Geomechanical modeling}

The subject of this study is the phenomenon known from the literature as "stress shadowing" which refers to the occurrence of altered $\mathrm{min} / \mathrm{max}$ horizontal stress ratio located in the area being under hydrocarbons exploitation. This phenomenon may lead to the changes in the direction of fracture propagation up to 90 degrees, generated after the period of production as a result of pore pressure decrease in the hydrocarbons depletion area.

In presented case the geomechanical modeling was performed with the use of combination of three Schlumberger software platforms accessible from Petrel 2013.2.

In this study authors considered a synthetic sandstone reservoir object with the width of 2000 feet and thickness of 50 feet. A shale layers, revealing near to zero permeability $(0,0001 \mathrm{mD})$ of 250 feet thickness each is laying below (light purple) and above (light green) the sandstone reservoir (Fig. 1).

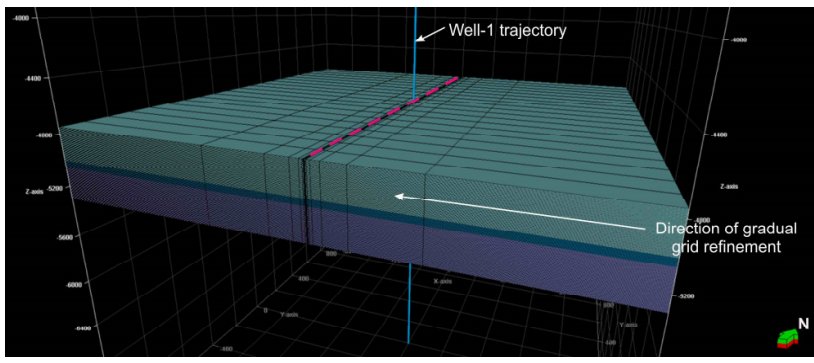

Figure 1 The geometry of the modeled reservoir object consisting of a sandstone layer (dark green) and a layer of shales above (light green) and below (light purple) the reservoir. In the center of the object a vertical oriented in the $N$-S direction fractures is located (marked with the pink dashed line). In the vicinity of the fracture a gradual grid refinement method was applied in order to increase the grid resolution in the area of hydrocarbons production.

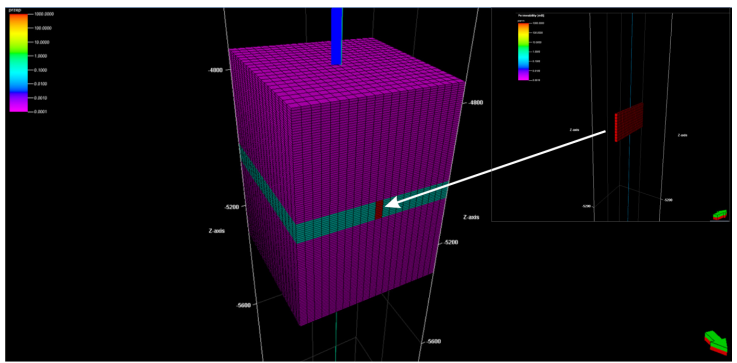

Figure 2 The geometry of the object with the presented distribution of permeability. Shale layers with near to zero permability $(0,0001$ $m D)$ are over-and underlying the sandstone reservoir with permeability of $0,1 \mathrm{mD}$. The central part of the reservoir a vertical fracture (zoomed in on the right side) is located with the permability of $1000 \mathrm{mD}$.

As an example of numerical simulation, a three variants of the initial ratio of the horizontal stress $\sigma \mathrm{H} /$ $\sigma \mathrm{h}$ acting on the reservoir, subjected to the geomechanical simulation were following: 1,01; 1,15 and 1,25 . 
The idea of the simulation is explained in details on the figure 3.

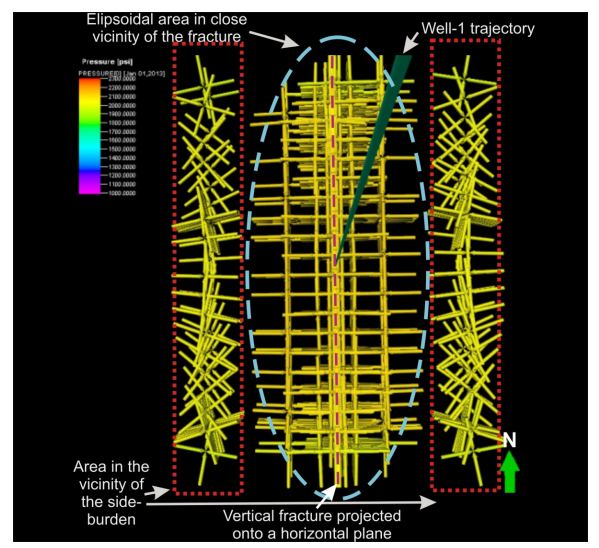

Figure 3 The zoom of the projection of analyzed object onto a horizontal plane perpendicular to the vertical axis consistent with the trajectory of the vertical well called Well-1. In the central part of the object a vertical fracture oriented to the $\mathrm{N}$ $S$ direction (which is consistent with the direction of $\sigma_{H}$ ) is marked with pink dashed line. The vicinity of producing fracture in the shape of ellipsoidal area is marked with blue dashed line. This area is expected to be especially subjected to the stress changes due to the hydrocarbons depletion. The area neighboring with the reservoir sideburden is marked with red dotted line. The apparent lack of symmetry on the image results from the projection angle. The vectors being the result of simulation (in this particular case - initial isotropic state of the reservoir is marked with the yellow tone color) indicate the real state of horizontal stresses (pressures) calculated from the data, showing both the direction of stresses and its magnitude according to the color bar (called tensor in the further description after Petrel manual).

\section{Conclusions}

- The concept and procedures within Mechanical Earth Model allow for visualizing the state of stresses and tracking the changes based on geologic, geophysics and reservoir engineering data.

- Decrease of the pore pressure due to ongoing production of hydrocarbons is the direct cause of change in state of stresses

- The best results refer to the refracturing of the initially isotropic medium. However the possibility of the return to the originally isotropic state of horizontal stresses of the reservoir which acquired anisotropic character during hydrocarbons exploitation cannot be excluded.

\section{Acknowledgements}

The Authors thank the Department of Geophysics at the Colorado School of Mines and prof. T.L.Davis for funding the fellowship during which the research was carried out.

Tom Bratton, Schlumberger Ambassador at the Colorado School of Mines, for scientific guidance and participation in discussions.

Schlumberger company for providing necessary software.

\section{References}

Dozier, G. [2003] Refracturing works. Oilfield Review. Houston, Texas, USA. Jack Elbel. Consultant. Dallas, Texas. Eugene Fielder. Devon Energy, pp. 38-53.

Dunphy, R. and Campagna, D. [2011] Fractures, Elastic Moduli \& Stress: Geological Controls on Hydraulic Fracture Geometry in the Horn River Basin. Conference Paper at Energy, Environment, Economy Recovery SCPG/CSEG/CWLS convention in Calgary, pp. 1-9.

Fjar, E., Holt, R.M., Raaen, A.M., Risnes and Horsrud, R.P. [2008] Petroleum Related Rock Mechanics, 2nd Edition. Elsevier.

Siebrits, E., Elbel, J.L., Hoover, R.S., Diyashev, I.R., Holditch-RT; Griffin, L.G., Demetrius, S.L., Wright, C.A., Davidson, B.M., Steinsberger, N.P. and Hill, D.G. [2000] Refracture Reorientation Enhances Gas Production in Barnett Shale Tight Gas Wells. SPE Annual Technical Conference and Exhibition held in Dallas, Texas, 1-4 October 2000. 